\title{
Quantitative and Statistics Analysis of Lineament from Landsat-8 OLI Imagery
}

\author{
Zhenjie Shi ${ }^{1,2, a}$, Xingping Wen ${ }^{1,2, b^{*}}$, Wei Ma ${ }^{1,2}$, Lijuan Zhang ${ }^{1,2}$, Jun Wang ${ }^{1,2}$, \\ Yang Zhou ${ }^{1,2}$
}

${ }^{1}$ Faculty of Land Resource Engineering, Kunming University of Science and Technology, Kunming, Yunnan, 650093, China

${ }^{2}$ Mineral Resources Prediction and Evaluation Engineering Laboratory of Yunnan Province, Kunming, Yunnan, 650093, China

aemail: shizhenjie8657@163.com, *Corresponding author ${ }^{b^{*}}$ email: wfxyp2008@gmail.com

Keywords: Huize Pb-Zn Deposit; Linear Structure; Quantitative Statistics; Direction

\begin{abstract}
Quantitative statistics analysis of lineament has been widely carried out in geological research, and Landsat data have been used most extensively for remote sensing geological investigations. This paper made an interpretation of lineament from Landsat-8 imagery and summarized the direction distributed characteristics based on regional geological background of the large scale Huize Pb-Zn ore deposit in Yunnan province of China. The research indicates that the predominant directions are NE and NW trending, the second dominant direction is nearly EW trending, and the weakest direction is NS trending. The result obtained in this research consistent with the regional directions and also with geological features of study area.
\end{abstract}

\section{Introduction}

Lineament is a variety of linear features presented in the aerial photo and satellite image, which reflects certain surface and hidden structural information. It helps geologists to gather information on the structural properties or to study regional fracture patterns that reveal some of the stress history[1]. The quantitative analysis of linear structure can be traced back to the 1970s, A.C Johnson(1974) compiled the linear azimuth deviation response surface diagram based on the remote image linear azimuth frequency statistics of Shelby Richland county, Ohio in the United States[2]. It is also researched and used for many Chinese scholars in the 1980s. They made a complete quantitative analysis method of lineaments for structural analysis and metallogenic prediction, and achieved good results in geological applications[3][4].

\section{Study Area and Geological Setting}

Huize $\mathrm{Pb}-\mathrm{Zn}$ ore deposit is the most famous production base of lead-zinc-germanium mine of China, located in south-central of the Sichuan-Yunnan-Guizhou Zn-Pb polymetallic metallogenic province. Its tectonic belonged to the southwestern margin of the Yangtze tectonic paraplatform and southern of northeast Yunnan depression basin. The Xiaojiang deep fault zone and the Qujing-Zhaotong concealed fault zone have provided favorable tectonic backgrounds for the formation of ore fluids and deposits. Huize Pb-Zn mining area is composed by Kuangshanchang, Qilinchang and Yinchangpo deposit. The stratum formed a two-tier structure gradually after a long time evolution and the ore bearing stratum is mainly Lower Carboniferous Baizuo Formation in cap rock.

The mining area is developed mainly by NE trending structure, represented by Kuangshanchang fault, Qilinchang fault and Yinchangpo fault that are the ore-guided structures of deposit. NE trending fractures laminated in layer are the main ore-host structures, which control the occurrence and shape of ore body. And NW trending fractures intersected with NE trending are the ore-distribution structures, which formed the Duozi-type structures are widespread ore-controlling structural types in the mining area[5]. 


\section{Preprocessing and Interpretation of RS Imagery}

This study uses the newer Landsat-8 OLI image(2014) whose orbit number is 129/42 and spatial resolution is $30 \mathrm{~m}$. Based on RapidEye image for OLI image geometric correction and fusion, supplemented by band combinations and spatial filtering will improve image's spatial resolution and the accuracy of interpretation. After processing, with color richness and texture clear, the image shows rich surface information for better interpretation. Based on understanding of geological background we get the linear structure figure through visual interpretation, but the roads, buildings and other artificial factors must be removed. Then the lineaments are considered as a result of geological processes and reflect the basic structural pattern of the study area[6].

\section{Azimuth Distributed Statistics of Lineament}

Analysis of Length and Frequency. Subjective factors of visual interpretation always affect the accuracy of linear structure interpretation. We need to count the length, frequency and cumulative length of linear structure for making the Length-Frequency and Length-Cumulative Length histograms. If the histogram shape obey the lognormal distribution, the interpretation is objective, reliable and in line with the general rules of Geology[2]. From the Fig.1, the shape of histogram, length(natural logarithm) as abscissa, frequency as ordinate, close to the lognormal distribution. Length-Cumulative Length histogram(Fig.2) is not only close to the lognormal distribution but also the Length-Frequency histogram. The histograms show that the length of linear structure in study area are randomly distributed, and the frequency present a high correlation with cumulative length in different interval. So the interpretation appears a high reliability and can be used as the basic data for follow quantitative analysis.

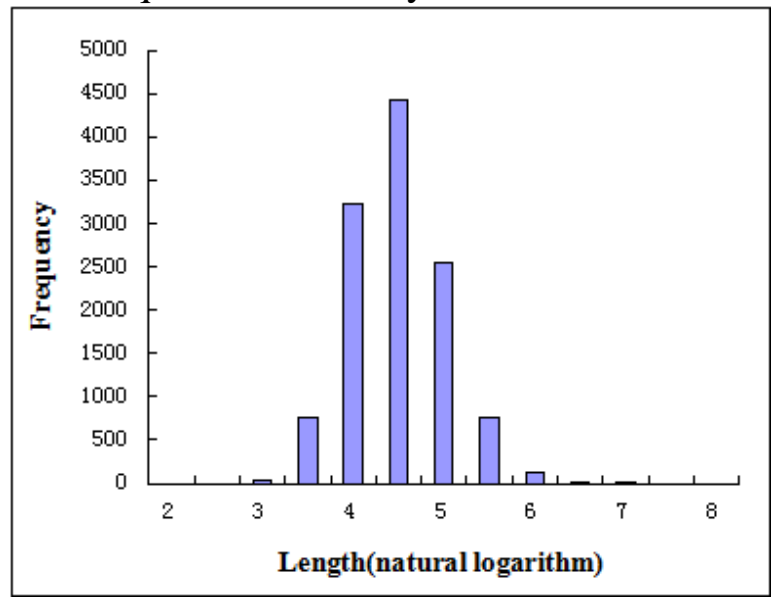

Fig.1. Length-Frequency histogram

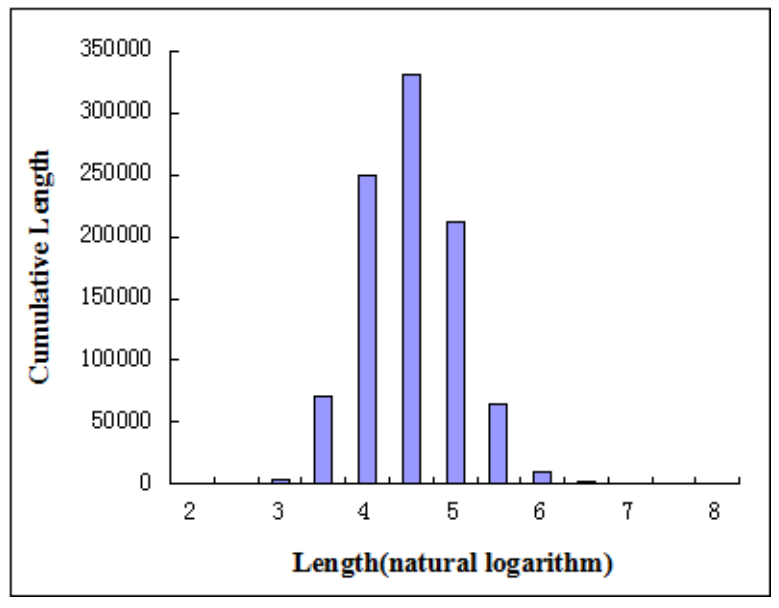

Fig.2. Length-Cumulative Length histogram

Analysis of Predominant Direction. Quantitative statistics of lineaments' trend are beneficial to analyze regional and partial geological structure information in the mining area. Then we can understand the structural pattern's contribution to ore-forming. Starting from $0^{\circ}$ and regarding $10^{\circ}$ as the intervals, counts the number and cumulative length of linear structure at every interval, and then gets the diagram(Fig.3, 4) and radar chart(Fig.5). From the diagram of Azimuth-Frequency, the curve distributes in a triple peak pattern, and the peak values locate at around $50^{\circ}, 100^{\circ}$ and $130^{\circ}$ respectively. The wave trough locates at $0^{\circ}$ or so. Azimuth-Cumulative Length diagram has the same shape. Therefore frequency and cumulative length present a high correlation too. The diagram shows that NE trending, NW trending and nearly EW trending are the predominant directions in Huize $\mathrm{Pb}-\mathrm{Zn}$ ore deposit, and SN trending is the disadvantages. The former are results of the regional tectonic evolution, also the favorable ore-controlling factors. The latter reflects the local structural anomalies, which is one of the factors considered for prospecting. Azimuth radar chart intuitively shows superiority trend and abnormal information of linear structure with different approach but equally satisfactory results and wonderful. 


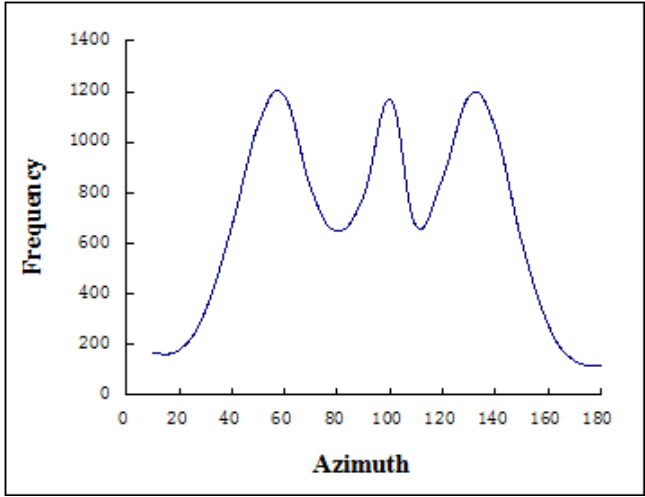

Fig.3. Azimuth-Frequency diagram

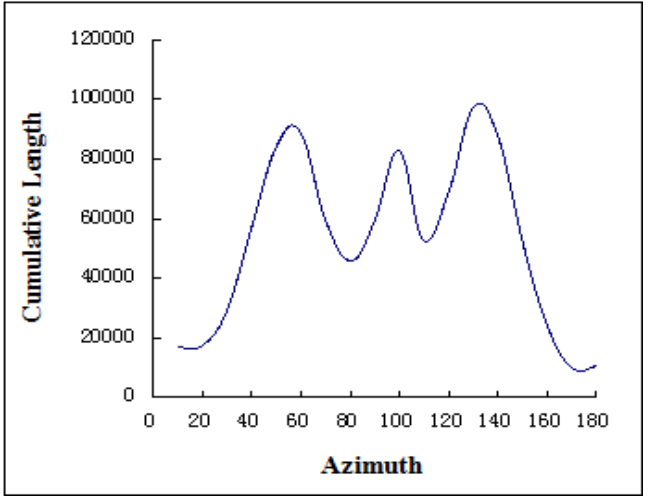

Fig.4. Azimuth-Cumulative Length diagram

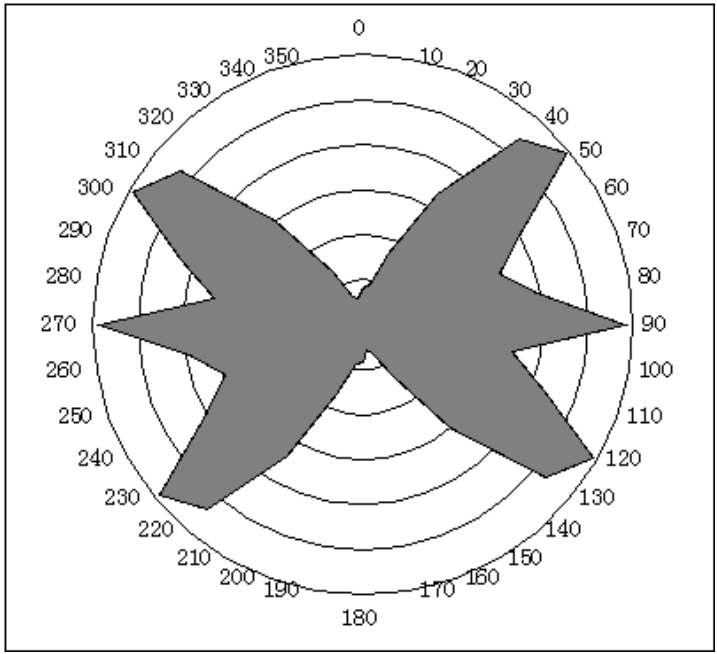

Fig.5. Azimuth radar chart

Statistical distribution curve always intuitively reflect the distribution rule of the random variable. In this paper, diagram with several peaks implies muti-period superposition of geological processes by a certain sequence[7]. In fact, NE structural zone is the uppermost ore-forming system of Huize $\mathrm{Pb}-\mathrm{Zn}$ ore deposit. Based on lineaments' interpretation from Landsat-8 and azimuth chart, SN structural zone has a small scale and intensity due to serious damage. NE and NW structural zone formed the typical Duozi-type ore-controlling structural pattern with obvious superiority azimuth. EW structural zone's scale is less than NE and NW, but it also has a salient orientation characteristic. For the reason that, EW trending should be a newer structural system developed in the Huize $\mathrm{Pb}-\mathrm{Zn}$ ore deposit.

\section{Discussions}

(1)Remote sensing linear structures are results of geological processes, controlled by geological stress fields, with certainty and randomicity. Therefore, the quantitative analysis of linear structure is an essential method for geological research.

(2)Azimuth statistics of linear structure in Huize Pb-Zn ore deposit appear that NE and NW trending structures are well-developed and have a direct relationship with ore-forming. As a newer structural system, EW trending has a significant feature on azimuth statistics. And SN trending structure developed weakly. All these are consistent with the actual geological situation of study area.

(3)The quantitative statistics analysis of linear structure, based on geological theory and in mathematical methods, can achieve better effects in analysis of regional tectonic pattern and other applications. This paper focus on studying of azimuth characteristics of linear structure in Huize $\mathrm{Pb}-\mathrm{Zn}$ ore deposit. The regional distribution rule of linear structure and ore-forming favorability will be carried out in-depth research in future work. 


\section{Acknowledgement}

This study was jointly supported by Natural Science Foundation of China (Grant No. 41101343), the remote sensing geochemistry disciplinary innovation team, Kunming University of Science and Technology, Kunming, China, 2013, and the innovation team of ore-forming dynamics and prediction of concealed deposits, Kunming University of Science and Technology, Kunming, China, 2008.

\section{References}

[1] Richard Lepage, Rouhana G. Rouhana, Benoît St-Onge, Rita Noumeir, Robert Desjardins. Cellular neural network for automated detection of geological lineaments on Radarsat images[J]. IEEE Transactions on Geoscience and Remote Sensing, 2000, 38(3): 1124

[2] JOHNSON A C. Lineament analysis: An exploration method for the delineation of structural and stratigraphic anomalies[C]. Proceedings of the First International Conference on the New Basement Tectonics, 1974. 449-452

[3] Buyi Zhao, Xiaoguang Qin. Quantitative analysis of remote sensing structure[J]. Geological Science and Technology Information, 1988, 7(1): 127-131(in Chinese)

[4] Runsheng Wang, Wenli Yang. Digital analysis of lineament field in remote sensing image[J]. Remote Sensing for Land \& Resources, 1992(3): 49-53(in Chinese)

[5] Runsheng Han, Jin Chen. Ore-controlling tectonics and prognosis of concealed ores in Huize Pb-Zn deposit[J]. Acta Mineralogica Sinica, 2001, 21(2): 255-266(in Chinese)

[6] Matthew J. Cracknell, Michael Roach, David Green, Arko Lucieer. Estimating Bedding Orientation From High-Resolution Digital Elevation Models[J]. IEEE Transactions on Geoscience and Remote Sensing, 2013, 51(5): 2949

[7] Shaoping Liu, Jun Tang, Xiaohong Xu. The methods and applications on mathematical geology[M]. Beijing: Petroleum industry press, 2011. 21-25(in Chinese) 